\title{
EXTENDED STRUCTURES IN THE PLANETARY NEBULAE HE2-111 AND HE2-119
}

\author{
J.A. LÓPEZ and M. TAPIA \\ Instituto de Astronomía, UNAM, Apartado Postal 877, Ensenada, Mexico \\ and \\ M. ROTH \\ Las Campanas Observatory, Casilla 601, La Serena, Chile
}

He 2-119 is a bright, elliptical, planetary nebula of relatively large size (117 arcsec along the major axis and 74 arcsec along the minor one). Digital, unsharp masking CCD imaging of this object is presented, revealing a filamentary, nearly bipolar inner structure. In addition, the discovery of an extended, faint halo in this object is reported. The halo has a diameter of 208 arcsec and has a nearly circular form. He 2-119 thus becomes a new member of the group of planetary nebulae with halos. Its general characteristics are discussed.

As for He 2-111 (the mandrill nebula) this is a planetary nebula best known for its core appearence, however, its structure is that of an extremely large bipolar (lobe size $\sim 5.4$ arcmin), probably produced by a Nova-like event. Deep CCD imaging is presented, showing most of its entire extent. As for the case of $\mathrm{He} 2-119$, digital, unsharp masking image processing techniques are used to display the bright core and the extended, faint lobes at levels that show the internal filamentary structure in both cases. A number of noteworthy morphological characteristics are pointed out in this most peculiar bipolar nebula.

The details of this work will be published in Rev. Mexicana Astron. Astrofis. 